\title{
WHY THERE CANNOT BE A SINGLE PROBABILISTIC MEASURE OF COHERENCE
}

\begin{abstract}
Bayesian Coherence Theory of Justification or, for short, Bayesian Coherentism, is characterized by two theses, viz. (i) that our degree of confidence in the content of a set of propositions is positively affected by the coherence of the set, and (ii) that coherence can be characterized in probabilistic terms. There has been a longstanding question of how to construct a measure of coherence. We will show that Bayesian Coherentism cannot rest on a single measure of coherence, but requires a vector whose components exhaustively characterize the coherence properties of the set. Our degree of confidence in the content of the information set is a function of the reliability of the sources and the components of the coherence vector. The components of this coherence vector are weakly but not strongly separable, which blocks the construction of a single coherence measure.
\end{abstract}

\section{INTRODUCTION}

Suppose that we have obtained various items of information from independent sources that are not fully reliable. Let an information set be a set containing such items of information. What does it mean to say that our degree of confidence is positively affected by the coherence of the information set? Certainly it need not be the case that coherence is the only determinant of our degree of confidence. For instance, the degree of confidence will also be determined by how reliable we take our information sources to be. Presumably there will be a range of sentences that fit the following schema:

(S) The greater $X$, the greater our degree of confidence will be that the content of the information set is true, ceteris paribus.

The ceteris paribus clause assumes that we keep all the other determinants of the degree of confidence of the information set fixed (cf. Bovens and Olsson, 2002). We will investigate what ought to be filled in for $X$, i.e. what the determinants are of our degree of confidence. It will turn out that one of these determinants is a reliability measure and the other determinants are various components of 
coherence, expressed in a vector of length $n$ for information sets of size $n$.

\section{NOTATION AND TECHNICAL PRELIMINARIES}

(i) Let $\mathrm{S}^{(n)}$ be an information set $\left\{\mathrm{R}_{1}, \mathrm{R}_{2}, \ldots, \mathrm{R}_{n}\right\}$. We construct propositional variables $\mathrm{R}_{1}, \mathrm{R}_{2}, \ldots, \mathrm{R}_{n}$ whose positive values are the propositions in the information set and whose negative values are their respective negations. In Figure 1, we represent a probability distribution over the propositional variables $R_{1}, R_{2}, R_{3}$. We introduce the parameters $a_{i}$ for $i=0, \ldots, n: a_{i}$ is the probability that $n-i$ of the propositional variables will take on positive values and $i$ propositional variables will take on negative values. Clearly, $\sum_{i=0}^{n} a_{i}=1$. We stipulate that the information is neither inconsistent nor certain, i.e. $a_{0} \in(0,1)$. Let's call $\left\langle a_{0}, \ldots, a_{n}\right\rangle$ the weight vector of the information set $\mathrm{S}^{(n)}$. Let $R E P R_{i}$ be the propositional variable whose positive value is that there is a report to the effect that $\mathrm{R}_{i}$ and whose negative value is that there is no report to the effect that $\mathrm{R}_{i}$.

(ii) When we are informed that some proposition is true, our source may be more or less reliable. Think of an information source as of a medical test that yields certain proportions for false positives and for false negatives. The reliability of an information source with

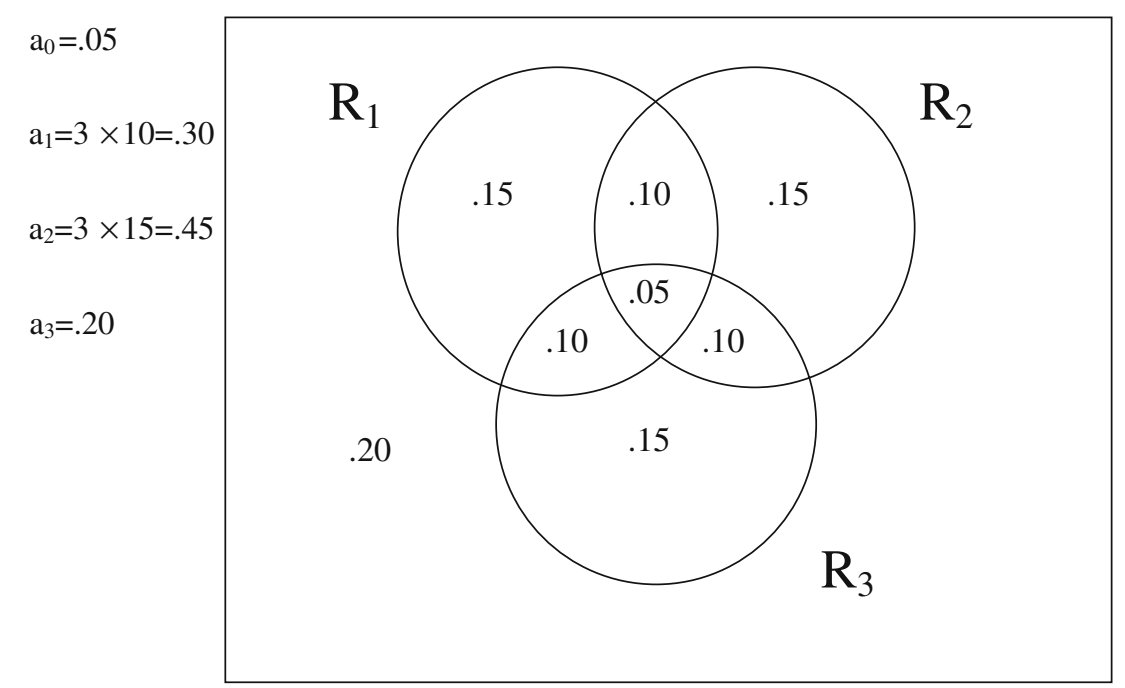

Figure 1. A diagram of the joint probability distribution over the variables $R_{1}, R_{2}$, and $R_{3}$. 
respect to the report in question ${ }^{1}$ can be readily expressed by the likelihood ratio

$$
x_{i}=\frac{q_{i}}{p_{i}} \text { for all sources } i=1, \ldots, n,
$$

in which $q_{i}=\mathrm{P}\left(\mathrm{REPR}_{i} \mid \neg \mathrm{R}_{i}\right)$ is the chance of a false positive and $1-p_{i}=1-\mathrm{P}\left(\mathrm{REPR}_{i} \mid \mathrm{R}_{i}\right)$ is the chance of a false negative. For an excellent medical test or information source, $x_{i}=0 / 1=0$, whereas for a test or source that is no better than random, $q_{i}=p_{i}$ and so $x_{i}=1$. Hence we propose $r_{i}=1-x_{i}$ as a measure of reliability. We exclude fully reliable and fully unreliable information sources: $r \in(0,1)$ and make the idealization that all sources are equally reliable, i.e. $r_{i}=r$ for all $i=1, \ldots, n .^{2}$

(iii) The coherence of an information set only affects our degree of confidence when the witnesses are at least to some degree independent. To keep things simple, let us assume that the witnesses are independent. Bovens and Olsson (2000) and Earman (2000) provide the following analysis of independence. To say that witnesses are independent is to say that each $R_{i}$ screens off the $R E P R_{i}$ from all $R_{j} \mathrm{~s}$ and all $R E P R_{j}$ s for $i \neq j$, i.e. $R E P R_{i}$ is probabilistically independent of all $R_{j}$ s and all $R E P R_{j}$ s given $R_{i}$. What this means in ordinary language is that the witnesses are not influenced by the reports of the others witnesses, nor by facts other than the fact that they are reporting on.

\section{INFORMATION SINGLETONS}

Let us first consider an information singleton. Suppose that we are informed by a less than fully reliable source that $\mathrm{R}$. What determines our degree of confidence that the information is true? This is just an application of Bayes Theorem. For notational convenience, let $\bar{\varphi}$ stand for $1-\varphi$ for any parameter $\varphi$.

$$
P^{*}(\mathrm{R})=P(\mathrm{R} \mid \mathrm{REPR})=\frac{a_{0}}{a_{0}+\overline{a_{0}} \bar{r}}
$$

Thereare two determinants to the degree of confidence for singletons, viz. $r$ and $a_{0} . a_{0}$ can be thought of as a measure of external coherence, i.e. a measure of how well the new item of information fits in with our background beliefs. Hence for information singletons, we can fill in the schema in (S): 
(Det $\left.{ }_{1}\right)$ The greater the reliability of the source, i.e. $r$, the greater our degree of confidence will be that the content of the information set is true, ceteris paribus.

$\left(\right.$ Det $\left._{2}\right)$ The greater the external coherence of the new item of information with our background beliefs, i.e $a_{0}$, the greater our degree of confidence will be that the content of the information set is true, ceteris paribus.

The ceteris paribus clause in (Det $)_{1}$ requires that we keep $a_{0}$ fixed, whereas in $\left(\right.$ Det $\left._{2}\right)$ it requires that we keep $r$ fixed. To show that these claims are true, we calculate the partial derivatives with respect to the respective measures of reliability and the measure of coherence:

$$
\begin{aligned}
& \frac{\partial P^{*}(\mathrm{R})}{\partial r}=\frac{\bar{r}}{a_{0}^{2}} P^{* 2}(\mathrm{R}) \\
& \frac{\partial P^{*}(\mathrm{R})}{\partial a_{0}}=\frac{\overline{a_{0}}}{a_{0}} P^{* 2}(\mathrm{R}) .
\end{aligned}
$$

Since $a_{0}, r, P^{*}(\mathrm{R}) \in(0,1)$, these partial derivatives are both positive, which confirms $\left(\right.$ Det $\left._{1}\right)$ and $\left(\right.$ Det $\left._{2}\right)$.

What seems somewhat trivial at this point, but will become highly relevant for larger information sets, is that $\left(\right.$ Det $\left._{1}\right)$ and $\left(\right.$ Det $\left._{2}\right)$ both need to be included in (S). Suppose that we would only include (Det $)$. Then the ceteris paribus claim would be vacuously true, since no other determinants are in play. But ( Det $_{1}$ ) by itself would be false: Certainly we could imagine that we would be more confident that a new item of information from a less reliable source is true than from a more reliable source, when this item has a much higher degree of external coherence, i.e. it fits in so much better with our background knowledge.

\section{INFORMATION PAIRS}

Let us now turn to information pairs. Suppose that we are informed by two independent and less than fully reliable sources that $R_{1}$ and $\mathrm{R}_{2}$, respectively. By applying Bayes Theorem and working out the independences, it can be shown ${ }^{3}$ that our degree of confidence that both $R_{1}$ and $R_{2}$ are true after receiving the items of information equals 


$$
\begin{aligned}
P^{*}\left(\mathrm{R}_{1}, \mathrm{R}_{2}\right) & :=P\left(\mathrm{R}_{1}, \mathrm{R}_{2} \mid \mathrm{REPR}_{1}, \mathrm{REPR}_{2}\right) \\
& =\frac{a_{0}}{a_{0}+a_{1} \bar{r}+a_{2} \bar{r}^{2}} \text { with } a_{2}=1-a_{0}-a_{1}
\end{aligned}
$$

There are three determinants to the degree of confidence for pairs, viz. $r$ and $a_{0}$ and the degree of internal coherence between $R_{1}$ and $R_{2}$. The internal coherence of $\mathrm{S}^{(2)}=\left\{R_{1}, R_{2}\right\}$ could be measured as follows:

$$
m\left(\mathrm{~S}^{(2)}\right)=\frac{P\left(\mathrm{R}_{1}, \mathrm{R}_{2}\right)}{P\left(\mathrm{R}_{1} \vee \mathrm{R}_{2}\right)}=\frac{a_{0}}{a_{0}+a_{1}}
$$

$m\left(\mathrm{~S}^{(2)}\right)$ measures the proportional overlap between $R_{1}$ and $R_{2}$ in the probability space. (This measure is suggested as a possible measure of coherence in Olsson (2002): 250). When we keep $a_{0}$ fixed, the measure increases as the marginal probabilities of $P\left(\mathrm{R}_{1}\right)$ and $P\left(\mathrm{R}_{2}\right)$ decrease, and hence when $\mathrm{R}_{1}$ and $\mathrm{R}_{2}$ become the more coherent. When $R_{1}$ and $R_{2}$ are minimally coherent, i.e. when they are mutually exclusive, then the measure equals 0 and when they are maximally coherent, i.e. when they are equivalent, the measure equals 1 .

We can now make a clear statement of the determinants of our degree of confidence for information pairs:

(Det $\left.{ }_{1}\right)$ The greater the reliability of the sources, i.e. $r$, the greater our degree of confidence will be that the content of the information set is true, ceteris paribus.

(Det $)_{2}$ The greater the external coherence of the new items of information, i.e $a_{0}$, the greater our degree of confidence will be that the content of the information set is true, ceteris paribus.

(Det $\left.{ }_{3}\right)$ The greater the internal coherence of the new items of information, i.e $m\left(\mathbf{S}^{(2)}\right)$, the greater our degree of confidence will be that the content of the information set is true, ceteris paribus.

These claims are all true: Following the standard procedure, the reader can easily verify that the partial derivatives of $P^{*}\left(\mathrm{R}_{1}, \mathrm{R}_{2}\right)$ with respect to the measures of reliability, external coherence and internal coherence are always positive. (The proof follows from a general proof that will be provided in Section 5.)

Furthermore, any proper subset of conditions $\left\{\left(\right.\right.$ Det $\left._{1}\right),\left(\right.$ Det $\left._{2}\right)$, $\left(\right.$ Det $\left.\left._{3}\right)\right\}$ fails to hold, because it restricts the reach of the ceteris paribus clause. The most interesting counterexample is a counter- 
example to the conditions $\left\{\left(\operatorname{Det}_{1}\right),\left(\operatorname{Det}_{3}\right)\right\}$. Since we do not include (Det $)_{2}$, there is no reason to keep $a_{0}$ fixed. Consider the following two information pairs. The information pairs $S$ and $S^{\prime}$ are characterized by the following vectors $\left\langle a_{0}, a_{1}, a_{2}\right\rangle=\langle .20, .70, .10\rangle$ and $\left\langle a_{0}^{\prime}, a_{1}^{\prime}, a_{2}^{\prime}\right\rangle=\langle .10, .10, .80\rangle$. We plot the posterior joint probabilities of $S$ and $S^{\prime}$ for different values of $r$ in Figure 2. Note that for some values of $r$, the posterior joint probability of $S$ exceeds the posterior joint probability of $S^{\prime}$, while for other values of $r$, the posterior joint probability of $S^{\prime}$ exceeds the posterior joint probability of $\mathrm{S}$. Hence, it is false to say that the reliability and the internal coherence are the relevant determinants of our degree of confidence. The ceteris paribus clause does not force us to keep the external coherence fixed, i.e. to set $a_{0}=a_{0}^{\prime}$. Figure 2 lets us make both a weaker and a stronger objection in response to the claim that the set $\left\{\left(\operatorname{Det}_{1}\right)\right.$, $\left(\right.$ Det $\left.\left._{3}\right)\right\}$ contains the determinants of our degree of confidence. The weaker objection is that it is false to say that the greater the internal coherence, as measured by $m(\mathbf{S})$, the greater our degree of confidence, ceteris paribus: $m\left(S^{\prime}\right)=1 / 2>2 / 9=m(\mathbf{S})$ and yet, for $r \in(0$, $2 / 3)$, the posterior joint probability of $S$ exceeds the posterior joint probability of $S^{\prime}$. The stronger objection is that it is false to say that the greater the internal coherence, as measured by any probabilistic measure, the greater our degree of confidence, ceteris paribus. A single measure of internal coherence will impose an ordering over $S$ and $S^{\prime}$, and yet for some values of $r$, the posterior joint probability of $S$ will exceed the posterior joint probability of $S^{\prime}$, while for other

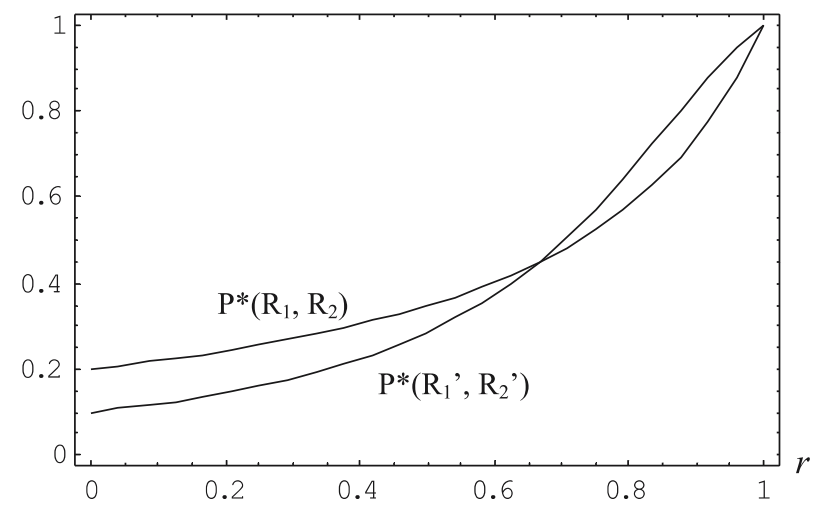

Figure 2. The posterior probability for information pairs with vectors $\left\langle a_{0}, a_{1}, a_{2}\right\rangle=\langle .20, .70, .10\rangle$ and $\left\langle a_{0}^{\prime}, a_{1}^{\prime}, a_{2}^{\prime}\right\rangle=\langle .10, .10, .80\rangle$ as a function of the reliability parameter $r$. 
values of $r$, the posterior joint probability of $S$ will exceed the posterior joint probability of $S^{\prime}$.

\section{GENERALIZING TO INFORMATION $N$-TUPLES}

One might be tempted to think that we have now found the determinants of our degrees of confidence, viz. reliability, external and internal coherence. The measure $m\left(\mathbf{S}^{(2)}\right)$ in (6) can be readily generalized to $m\left(\mathbf{S}^{(n)}\right)$ :

$$
m\left(\mathrm{~S}^{(n)}\right)=\frac{P\left(\mathrm{R}_{1}, \ldots, \mathrm{R}_{n}\right)}{P\left(\mathrm{R}_{1} \vee \ldots \vee \mathrm{R}_{n}\right)}=\frac{a_{0}}{\sum_{i=0}^{n-1} a_{i}}=\frac{a_{0}}{1-a_{n}}
$$

Furthermore, it is easy to show ${ }^{4}$ that the formula in (5) generalizes to

$$
P\left(\mathrm{R}_{1}, \ldots, \mathrm{R}_{n} \mid \mathrm{REPR}_{1}, \ldots, \mathrm{REPR}_{n}\right)=\frac{a_{0}}{\sum_{i=0}^{n} a_{i} \bar{r}^{i}} .
$$

We can now rephrase $\left(\operatorname{Det}_{3}\right)$ :

$\left(\operatorname{Det}_{3}^{\prime}\right)$ The greater the internal coherence of the new items of information, i.e $m\left(\mathbf{S}^{(n)}\right)$, the greater our degree of confidence will be that the content of the information set is true, ceteris paribus.

$\left\{\left(\operatorname{Det}_{1}\right),\left(\operatorname{Det}_{2}\right),\left(\operatorname{Det}_{3}^{\prime}\right)\right\}$ is then the set of all determinants of our degree of confidence.

This turns out to be a mistake. To see that this is a mistake, let us assume for a second that this were true for information triples. To show that $\left\{\left(\operatorname{Det}_{1}\right),\left(\operatorname{Det}_{2}\right),\left(\operatorname{Det}_{3}^{\prime}\right)\right\}$ does not hold for information triples, pick any two triples $\mathrm{S}$ and $\mathrm{S}^{\prime}$ with probability distributions so that $\left\langle a_{0}, a_{1}, a_{2}, a_{3}\right\rangle=\langle .05, .3, .1, .55\rangle$ and $\left\langle a_{0}^{\prime}, a_{1}^{\prime}, a_{2}^{\prime}, a_{3}^{\prime}\right\rangle=$ $\langle .05, .2, .7, .05\rangle$. Notice that the external coherence of both information sets is held fixed, i.e. $a_{0}=a_{0}^{\prime}$. We plot the posterior probability of these two information sets for different values of $r$ in Figure 3. Again, we can make a weaker objection and a stronger objection. The weaker objection is that it is false to say that the greater the internal coherence, as measured by $m(\mathbf{S})$, the greater our degree of confidence, ceteris paribus: $m(\mathrm{~S})=.05 / .45>.05 / .95=m\left(\mathrm{~S}^{\prime}\right)$ and yet, for $r \in(.8,1)$, the posterior joint probability of $\mathrm{S}^{\prime}$ exceeds the posterior joint probability of $\mathrm{S}$. The stronger objection is that it is false to say 


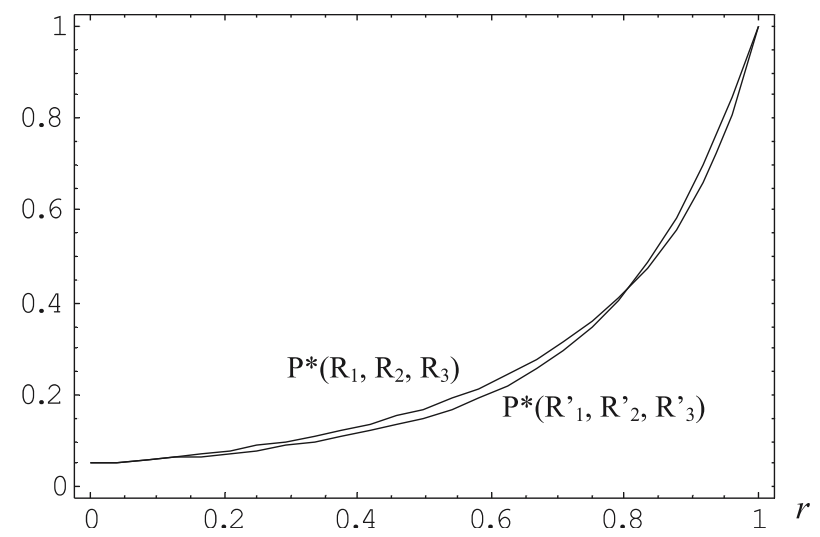

Figure 3. The posterior probability for information triples with weight vectors $\left\langle a_{0}\right.$, $\left.a_{1}, a_{2}, a_{3}\right\rangle=\langle .05, .3, .1, .55\rangle$ and $\left\langle a_{0}^{\prime}, a_{1}^{\prime}, a_{2}^{\prime}, a_{3}^{\prime}\right\rangle=\langle .05, .2, .7, .05\rangle$ as a function of the reliability parameter $r$.

that the greater the internal coherence, as measured by any probabilistic measure, the greater our degree of confidence, ceteris paribus. A measure of internal coherence will impose an ordering over $\mathbf{S}$ and $\mathbf{S}^{\prime}$, and yet for some values of $r$, the posterior joint probability of $\mathrm{S}$ will exceed the posterior joint probability of $\mathrm{S}^{\prime}$, while for other values of $r$, the posterior joint probability of $\mathrm{S}$ will exceed the posterior joint probability of $\mathrm{S}^{\prime}$.

So what can be done for information triples? Note that for information singletons, there is a unary vector of coherence determinants of the posterior probability, viz. $\left\langle a_{0}\right\rangle$; for information pairs there is a binary vector of coherence determinants, viz. $\left\langle a_{0} /\left(a_{0}+a_{1}\right)\right.$, $\left.a_{0}\right\rangle$. We can make the following generalization. The vector of coherence determinants for information $n$-tuples contains the following $n$ components: the ratio of the joint probability $a_{0}$ over the probability that at least $i$ of the $n$ propositions are true for $i=n-1$, this same ratio for $i=n-2, \ldots$, this same ratio for $i=0$. For singletons, this is the vector $\left\langle a_{0}\right\rangle$. For pairs this is the vector $\left\langle a_{0} /\left(a_{0}+a_{1}\right), a_{0}\right\rangle$. For triples, this is the vector $\left\langle a_{0} /\left(a_{0}+a_{1}\right), a_{0} /\left(a_{0}+a_{1}+a_{2}\right), a_{0}\right\rangle$. For $n$-tuples, this is the vector $\left\langle a_{0} /\left(a_{0}+a_{1}\right), \ldots, a_{0} /\left(a_{0}+a_{1}+\ldots+a_{n-1}\right)\right.$, $\left.a_{0}\right\rangle$. This can be represented by means of the following shorthand. Let

(9) $\quad c_{k}=\frac{a_{0}}{\sum_{i=0}^{k} a_{i}}$ for $k=1, \ldots, n$. 
We can define a vector that contains the internal coherence determinants,

$$
\vec{c}^{\text {int }}=\left\langle c_{1}, \ldots, c_{n-1}\right\rangle,
$$

and an encompassing vector that contains both the internal coherence determinants and the external coherence determinant $c^{\mathrm{ext}}=c_{n}=a_{0}$ :

$$
\vec{c}=\left\langle c_{1}, \ldots, c_{n}\right\rangle=\left\langle\vec{c}^{\mathrm{int}}, c^{\mathrm{ext}}\right\rangle
$$

Does this generalization for $n$-tuples hold? We will follow the standard procedure and show that the partial derivatives of $P^{*}\left(R_{1}, \ldots\right.$, $R_{n}$ ) with respect to $r$, and all $c_{k}$ s (for $\left.k=1, \ldots, n\right)$ are all positive. First we present a representation of $P^{*}\left(R_{1}, \ldots, R_{n}\right)$ in (12) that is more convenient to calculate the partial derivatives (proof omitted):

$$
P^{*}\left(\mathrm{R}_{1}, \ldots, \mathrm{R}_{n}\right)=\frac{1}{r \sum_{i=0}^{n-1} \frac{\bar{T}^{i}}{c_{i}}+\frac{r^{n}}{c_{n}}}
$$

We calculate the partial derivatives:

$$
\begin{aligned}
& \frac{\partial P^{*}\left(\mathrm{R}_{1}, \ldots, \mathrm{R}_{n}\right)}{\partial r}=\sum_{i=1}^{n} i\left(\frac{1}{c_{i}}-\frac{1}{c_{i-1}}\right) \bar{r}^{i-1} P^{* 2}\left(\mathrm{R}_{1}, \ldots, \mathrm{R}_{n}\right) \\
& \frac{\partial P^{*}\left(\mathrm{R}_{1}, \ldots, \mathrm{R}_{n}\right)}{\partial c_{n}}=\frac{\bar{r}^{n}}{c_{n}^{2}} P^{* 2}\left(\mathrm{R}_{1}, \ldots, \mathrm{R}_{n}\right) \\
& \frac{\partial P^{*}\left(\mathrm{R}_{1}, \ldots, \mathrm{R}_{n}\right)}{\partial c_{k}}=\frac{r \bar{r}^{k}}{c_{k}^{2}} P^{* 2}\left(\mathrm{R}_{1}, \ldots, \mathrm{R}_{n}\right) \text { for } k=1, \ldots, n-1
\end{aligned}
$$

Since $r, P^{*}\left(\mathrm{R}_{1}, \ldots, \mathrm{R}_{n}\right) \in(0,1)$ and $c_{i}<c_{i-1}$ for $i=1, \ldots, n$, these expressions are all greater than 0 . This confirms that the following are the determinants for our degree of confidence that the content of an information $n$-tuple is true are $\left(\operatorname{Det}_{1}\right),\left(\operatorname{Det}_{2}\right)$ and for all $i=1, \ldots, n-1$,

$\left(\operatorname{Det}_{2+i}\right)$ The greater the component of the internal coherence of the new items of information that is measured by $c_{i}$, the greater our 
degree of confidence will be that the content of the information set is true, ceteris paribus.

Note that $c_{k}=P\left(\mathrm{R}_{1}, \ldots, \mathrm{R}_{n} \mid\right.$ at least $n-k$ propositions true). Note further that an expression similar to eq. (15) holds if $c_{k}$ is replaced by $t_{k}=c_{k} / a_{0} . t_{k}$ is the ratio measure which is a common way to measure how well the information set $\left\{\mathrm{R}_{1}, \ldots, \mathrm{R}_{n}\right\}$ is supported by the evidence that at least $n-k$ propositions are true. Dietrich and Moretti (forthcoming) have already pointed out that there is an interesting relation between coherence and confirmation. We plan to elaborate on this in future work in the context of our criterion for a coherence quasi-ordering (Bovens and Hartmann: 2003a, b).

\section{SEPARABILITY}

So where did things go wrong in the attempt to measure coherence? There seems to be a focus on finding a single measure of coherence. This is a mistake. First, we need to have both external and internal measures of coherence, already when the information sets that are being compared are just pairs. One might argue that an external coherence measure is really not a coherence measure but rather a measure of how plausible or expected the new information is. ${ }^{5}$ But this is just a verbal dispute and it is certainly not entirely implausible to say that $a_{0}$ is a coherence measure, because it measures how well the new information coheres with our background beliefs. What is important is that we can characterize $a_{0}$, which we have dubbed "a measure of external coherence", as the last entry in a vector of measures that are governed by a common mathematical structure. Second, as the information set grows, we need multiple complementary internal measures of coherence. The posterior probability of the information set of size $n$ is determined by a reliability measure and a vector of $n$ coherence determinants.

Borrowing from preference theory, there is a very concise way of spelling out the point that we have made in this paper, viz. the probabilistic determinants of our degrees of confidence are weakly, but not strongly separable. What does this mean? ${ }^{6}$ Let us construct a simple illustration in preference theory. Suppose that I have two baskets with wine, vodka and cheese. When my preferences are weakly separable, then the following holds:

(Weak Separability) Take any two pairs of baskets $\left\{B_{1}, B_{2}\right\}$ and $\left\{B_{1}^{\prime}\right.$, $\left.\mathrm{B}_{2}^{\prime}\right\}$. For all commodities $i$, the following holds true. Let $\mathrm{B}_{1}$ and $\mathrm{B}_{1}^{\prime}$ 
contain the same amount of a particular commodity $i$ and similarly for $\mathrm{B}_{2}$ and $\mathrm{B}_{2}^{\prime}$. Furthermore, let $\mathrm{B}_{1}$ and $\mathrm{B}_{2}$ contain the same amounts of all other commodities $j \neq i$ and similarly for $\mathrm{B}_{1}^{\prime}$ and $\mathrm{B}_{2}^{\prime}$. Then $\mathrm{B}_{1} \succ \mathrm{B}_{2}$ iff $\mathrm{B}_{1}^{\prime} \succ \mathrm{B}_{2}^{\prime}$.

When my preferences are strongly separable, then the following holds true:

(Strong Separability) Take any two pairs of baskets $\left\{B_{1}, B_{2}\right\}$ and $\left\{B_{1}^{\prime}\right.$, $\left.\mathrm{B}_{2}^{\prime}\right\}$. Let $C$ be the set of types of commodities. For all proper subsets of types of commodities $c \subset C$, the following holds true. Let $B_{1}$ and $B_{1}^{\prime}$ contain the same amounts of all types of commodities in a particular $c$ and similarly for $\mathrm{B}_{2}$ and $\mathrm{B}_{2}^{\prime}$. Furthermore, let $\mathrm{B}_{1}$ and $\mathrm{B}_{2}$ contain the same amounts of all types of commodities in the complement of $c$ and similarly for $\mathrm{B}_{1}^{\prime}$ and $\mathrm{B}_{2}^{\prime}$. Then $\mathrm{B}_{1} \succ \mathrm{B}_{2}$ iff $\mathrm{B}_{1}^{\prime} \succ \mathrm{B}_{2}^{\prime}$.

How is it that my preferences over commodities could be weakly but not strongly separable? To be weakly separable it is sufficient that if one basket contains more of some commodity than another basket, ceteris paribus (i.e. keeping the amounts of all other commodities fixed), then I prefer the former to the latter. If there is no distinction between the amounts of any of the other items, then a basket with more wine is better than a basket with less wine. And similarly for vodka and for cheese. But strong separability imposes a stronger requirement. Let $c$ be the commodities wine and vodka. Let $\mathrm{B}_{1}$ and $\mathrm{B}_{1}^{\prime}$ contain the same amounts of wine and vodka, viz. lots of wine and little vodka, and let $\mathrm{B}_{2}$ and $\mathrm{B}_{2}^{\prime}$ contain the same amounts of wine and vodka, viz. lots of vodka and little wine. Furthermore, let $B_{1}$ and $\mathrm{B}_{2}$ contain the same amounts of cheese, viz. lots and let $\mathrm{B}_{1}^{\prime}$ and $\mathrm{B}_{2}^{\prime}$ contain the same amounts of cheese, viz. little. Considering that wine and cheese mix better than wine and vodka, one might expect that $\mathrm{B}_{1} \succ \mathrm{B}_{2}$ and $\mathrm{B}_{2}^{\prime} \succ \mathrm{B}_{1}^{\prime}$. This is a violation of strong separability.

When our preferences are weakly separable, we can construct utility functions $u_{i}$ over each commodity so that our preferences over baskets can be expressed by a function $U$ of the utility functions over each commodity. In addition, Debreu (1960) (as cited in Broome, 1991: 70) has shown that there exist functions $u_{i}$ so that $U$ is an additive function if and only if our preferences are also strongly separable. So if our preferences are strongly separable, then we could construct a preference ordering over the baskets only with respect to their liquid content - i.e. their content of wine and vodka: We could simply take the sum of the additive utility values for wine and vodka 
to reflect such an ordering. But this is not possible when our preferences are only weakly separable: There will be some pairs of baskets which we cannot order with respect to their liquid content, since the direction of the ordering is contingent on the amount of cheese.

Let us now turn back to the probabilistic determinants of our degrees of confidence. Certainly these determinants are weakly separable: The partial derivatives show that if one information set scores higher on some determinant than another information set, ceteris paribus - i.e. keeping all the other determinants fixed - then the posterior probability of the former will be higher than of the latter. What our counter examples show is that strong separability between these measures does not hold. To see this, consider the pairs of information sets $\mathrm{S}$ and $\mathrm{S}^{\prime}$ with their associated weight vectors $\left\langle a_{0}, \ldots, a_{n}\right\rangle$ and $\left\langle a_{0}^{\prime}, \ldots, a_{n}^{\prime}\right\rangle$ in our counter examples. It was not possible to order these vectors so that $P^{*}\left(\mathrm{R}_{1}, \ldots, \mathrm{R}_{n}\right)$ is greater than $P^{*}\left(\mathrm{R}_{1}^{\prime}, \ldots, \mathrm{R}_{n}^{\prime}\right)$ (or vice versa) for all values of $r$. Hence, it is impossible to order the coherence vectors $\left\langle a_{0} /\left(a_{0}+a_{1}\right), \ldots, a_{0} /\left(a_{0}+a_{1}+\ldots\right.\right.$ $\left.\left.+a_{n-1}\right), a_{0}\right\rangle$ and so that $P^{*}\left(\mathrm{R}_{1}, \ldots, \mathrm{R}_{n}\right)$ is greater than $P^{*}\left(\mathrm{R}_{1}^{\prime}, \ldots \mathrm{R}_{n}^{\prime}\right)$ (or vice versa) for all values of $r$. This is a violation of strong separability.

Let us return to our example from economics now to see what the lack of strong separability means for our assessment of coherence. If my preferences over commodities are not strongly separable, then there are certain pairs of baskets that I cannot order according to their liquid content, ceteris paribus - i.e. under the assumption that their cheese contents are held fixed: It depends on the amount of cheese in these baskets whether I will prefer one basket over the other. We have shown that the determinants of my degree of confidence in the content of an information set are not strongly separable. If coherence is the property of an information set that increases my degree of confidence in the content of the information set, ceteris paribus, then the following holds. There are certain pairs of information sets that I cannot order according to their coherence. Some pairs are such that my degree of confidence in one information set will be greater for some degrees of reliability, while my degree of confidence in the other information set will be greater for other degrees of reliability.

On the other hand, there are certain pairs of baskets which I can order according to their liquid content: I don't need to see how much cheese there is to know that I prefer the basket with lots of wine and vodka to the basket with little wine and vodka, as long as both 
baskets contain the same amounts of cheese. Similarly, there are certain pairs of information sets $\mathrm{S}$ and $\mathrm{S}^{\prime}$ that I can order according to which one is more coherent: I don't need to know how reliable the informers are to know that my degree of confidence in the content of $\mathrm{S}$ will exceed my degree of confidence in the content of $\mathrm{S}^{\prime}$, as long as they are equally reliable. Hence, just like we can construct a quasiordering over the baskets according to their liquid content, we can construct a quasi-ordering over the information sets according to their coherence. Under what conditions we can and cannot impose an ordering on a pair of information sets is an interesting question, but beyond the scope of this paper. ${ }^{7,8}$

\section{NOTES}

1 This is different from the reliability of an information source tout court. To see this distinction consider the case in which $q=0$. In this case, $r$ will reach its maximal value, no matter what the value of $p$ is. Certainly a source that provides fewer rather than more false negatives, as measured by $1-p$, is a more reliable source tout court. But when $q$ is 0 , the reliability with respect to the report in question is not affected by the value of $p>0$. No matter what the value of $p$ is, we can be fully confident that what the source says is true, since $q=0$ - i.e. the source never provides any false positives. When we speak of the reliability of the sources, we will mean the reliability of the source with respect to the report in question, rather than the reliability of the source tout court.

${ }^{2}$ For a justification of the assumption of equal reliability in determining a measure of coherence, see Bovens and Hartmann (2003b: 45-47).

3 The proof is straightforward: Apply Bayes Theorem; simplify on grounds of the independences in the screening off condition and substitute in the parameters $p$ and $q$; divide numerator and denominator by $p^{2}$; substitute in the parameters $r$ and $a_{i}$ for $i=0,1$, and 2 .

${ }^{4}$ See Bovens and Hartmann (2003a: 607-10 and 2003b: 131-133).

5 See Bovens and Hartmann (2003a: 605 and b: 10).

${ }^{6}$ For an introduction to separability, see Broome (1991: 60-89).

7 Note that our procedure is different than in Bovens and Hartmann (2003a, b) and that it will not yield the same coherence quasi-ordering. The reason is that we conceive of coherence here as covering both external and internal coherence, whereas, in Bovens and Hartmann (2003a: 605-606 and b: 10-11), we distinguish between the expectedness of the information - which corresponds to the external coherence - and the coherence - which corresponds to the internal coherence. The term coherence is ambiguous in ordinary language. Suppose that one is told that AIDS is caused by malnutrition and that AIDS is due to a vitamin-B deficiency. Is this information coherent? Well, yes and no. Yes, because one might say that, though the information is implausible, given my background knowledge about AIDS, this does not stand in the way of proclaiming that the information is coherent. This is the notion of coherence that is analyzed in (2003a, 2003b). No, because one might say that this information coheres very poorly with one's background knowledge. 
Granted, the information items cohere well between themselves, but this internal coherence is not sufficient to make us proclaim that the information is coherent. This is the notion that is analyzed in this paper.

${ }^{8}$ We are grateful for the support of the Alexander von Humboldt Foundation, the Federal Ministry of Education and Research and the Program for the Investment in the Future (ZIP) of the German Government through a Sofja Kovalevskaja Award.

\section{REFERENCES}

Bovens, L. and S. Hartmann: 2003a, 'Solving the Riddle of Coherence', Mind 112, 601-634.

Bovens, L. and S. Hartmann: 2003b, Bayesian Epistemology, Oxford University Press, Oxford.

Bovens, L. and E. J. Olsson: 2000, 'Coherentism, Reliability and Bayesian Networks', Mind 109, 685-719.

Bovens, L. and E. J. Olsson: 2002, 'Believing More, Risking Less - On Coherence, Truth and Non-Trivial Extensions', Erkenntnis 52, 137-150.

Broome, J.: 1991, Weighing Goods, Blackwell, Oxford.

Debreu, G.: 1960, 'Topological Methods in Cardinal Utility Theory', in Kenneth J. Arrow, Samuel Karlin and Patrick Suppes (eds.): 1959, Mathematical Methods in the Social Sciences 1959: Proceedings of the First Stanford Symposium. Stanford University Press, Stanford, pp. 16-26.

Earman, J.: 2000, Hume's Abject Failure, Oxford University Press, Oxford.

Dietrich, F. and L. Moretti: 2006, 'On Coherent Sets and the Transmission of Confirmation', Philosophy of Science 72(3), 403-424.

Olsson, E. J.: 2001, 'Why Coherence is not Truth-Conducive', Analysis 61, 186-193.

Olsson, E. J.: 2002, 'What is the Problem of Coherence and Truth?', Journal of Philosophy 94, 246-272.

Department of Philosophy,

Logic, and Scientific Method

London School of Economics and Political Science

Houghton Street

London, WC2A 2AE, UK

E-mail: L.Bovens@LSE.ac.uk

E-mail: S.Hartmann@LSE.ac.uk 